\title{
On the Genesis of the Woods Hole Fixed Point Theorem
}

\author{
Loring $W . T u$
}

The Woods Hole fixed point theorem is a farreaching extension of the classical Lefschetz fixed point theorem to vector bundles. It has as corollaries a holomorphic Lefschetz formula for complex manifolds and the Weyl character formula for the irreducible representations of a compact Lie group. Apart from its importance in its own right, the Woods Hole fixed point theorem is crucial in the history of mathematics as a precursor to the Atiyah-Bott fixed point theorem for elliptic complexes [7], one of the crowning glories of the analysis and topology of manifolds. On the algebraic side it led to Verdier's Lefschetz fixed point theorem in étale cohomology ([13], [23]). Indeed, Atiyah was awarded the Fields Medal in 1966 and the citation reads in part that Atiyah "proved jointly with Singer the index theorem of elliptic operators on complex manifolds" and "worked in collaboration with Bott to prove a fixed point theorem related to the Lefschetz formula." The discovery of these fixed point theorems dates back to the AMS Summer Research Institute on Algebraic Geometry at Woods Hole, a small town by the sea in Massachusetts, in 1964. With the passage of time, memories of how the theorems came about have become somewhat murky. In 2001 Raoul Bott unwittingly ignited a controversy through an interview published in the Notices of the AMS, a controversy that to this day has not been settled.

There are three protagonists in this drama: Michael Atiyah, Raoul Bott, and Goro Shimuraall three giants of twentieth-century mathematics.

Loring $W . T u$ is professor of mathematics at Tufts University. His email address is 1oring. tu@tufts . edu.

For permission to reprint this article, please contact: reprint-permission@ams .org.

DOI: http://dx.doi.org/10.1090/noti1284
Atiyah, best known for the index theorem, has made extensive and deep contributions to analysis, topology, K-theory, and mathematical physics. Bott, through foundational work in topology, had a profound influence on geometry and topology in the last sixty years. Shimura, known among other things for the Shimura variety, played a key role in the proof of Fermat's Last Theorem through his formulating the Shimura-Taniyama conjecture. Each of them has written a recollection of the genesis of the Woods Hole fixed point theorem ([1], [10], [11], [15], [16], [17]). The controversy centers on Shimura's exact contribution.

While editing Volume 5 of Bott's Collected Papers, I wrote to both Atiyah and Shimura for commentaries. In the process I discovered that there is some unfinished business. Sometimes history is best written not by the participants but by an unbiased observer. I have no stake in the outcome and my purpose is to examine the written record, to interview the participants, and to summarize the divergent points of view. It may not be possible to arrive at the historical truth acceptable to all but, by publishing this account in the Notices, perhaps one can inch one step closer. Unfortunately, Raoul Bott is no longer with us, but he did leave behind a voluminous written record, which forms part of the basis of my inquiry. In the interest of full disclosure, I should mention that Goro Shimura was my undergraduate thesis advisor at Princeton and Raoul Bott was one of my mentors at Harvard and that I owe them both a debt of gratitude.

The Classical Lefschetz Fixed Point Theorem The classical Lefschetz fixed point theorem is the beautiful statement that the number of fixed points of a nondegenerate smooth map $f: M \rightarrow M$ 


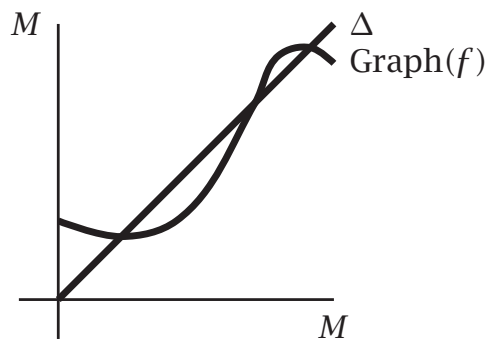

Figure 1. A transversal intersection of $\mathrm{Graph}(f)$ with the diagonal.

from a compact oriented manifold $M$ to itself is the Lefschetz number of $f$, i.e., the alternating sum $\left.\sum_{q}(-1)^{q} \operatorname{tr} f^{*}\right|_{H^{q}(M ; \mathbb{R})}$ of the traces of the induced linear maps on the real cohomology vector spaces $H^{q}(M ; \mathbb{R})$. Here "nondegenerate" means that the graph Graph $(f)$ of $f$ meets the diagonal $\Delta$ of $M \times M$ transversely and that fixed points are counted with multiplicities \pm 1 , as is usual in intersection theory, depending on the orientations of $\operatorname{Graph}(f)$ and $\Delta$.

For example, if $f: S^{2} \rightarrow S^{2}$ is the rotation of the sphere through $\alpha$ radians about the $z$-axis, for $0<\alpha<2 \pi$, then $f$ has two fixed points, the north and the south poles, each counted with multiplicity 1 . The cohomology vector spaces $H^{q}\left(S^{2} ; \mathbb{R}\right)$ of the 2 -sphere are $\mathbb{R}, 0, \mathbb{R}$ in dimensions 0,1 , and 2 respectively. It is easy to see that the induced maps $f^{*}$ on $H^{q}\left(S^{2} ; \mathbb{R}\right)$ are $\mathbb{1}$ (the identity map), 0 , and $\mathbb{1}$ respectively, so the Lefschetz number of $f$ is

$$
L(f)=1-0+1=2,
$$

which is the number of fixed points (Figure 2).

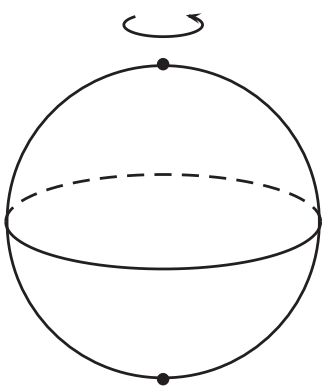

Figure 2. A rotation of the sphere about the $z$-axis has two fixed points. The Lefschetz number of the rotation is also 2 .

The Lefschetz fixed point theorem has some stunning consequences. For one thing, it implies quite easily the Hopf index theorem for vector fields with nondegenerate zeros: if a smooth vector field on a compact oriented manifold has nondegenerate zeros, then the total number of zeros, counted with multiplicity, is the Euler characteristic of the manifold (Figure 3). As another consequence, if the manifold $M$ is contractible,

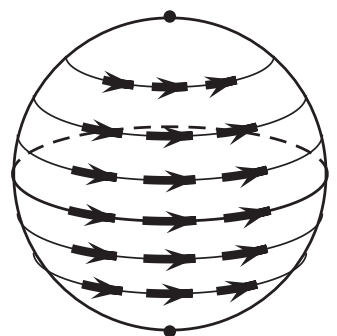

Figure 3. This vector field on the sphere has two zeros, each with multiplicity 1 . The Euler characteristic of the sphere is also 2 .

then its cohomology vanishes in positive dimensions and $H^{0}(M ; \mathbb{R})=\mathbb{R}$ in dimension 0 . Thus the induced map $f^{*}$ on cohomology is the identity map in $H^{0}$ and is otherwise the zero map, so that the Lefschetz number of $f$ is 1 . In this way the Lefschetz fixed point theorem implies that every smooth map from a contractible manifold to itself has a fixed point. This is the smooth analog of Brouwer's fixed point theorem for a continuous map of a disk.

\section{The Controversy}

At one point in his 2001 Notices interview with Allyn Jackson [9], Raoul Bott spoke about working with Michael Atiyah at the 1964 Woods Hole conference. He said, "During the conference we discovered our fixed point theorem, the Lefschetz fixed point theorem in this new context. This was a very pleasant insight. The number theorists at first told us we must be wrong, but then we turned out to be right. So we enjoyed that!" [9, p. 379]. In a letter to the editor [15], Shimura bridled at the description of the discovery, which made no mention of Shimura's conjecture. He also objected to Bott's memory that some number theorists were wrong, interpreting it as an intentional putdown of number theorists, saying that he was not aware of any number theorist who was against his conjecture.

In a gracious mea culpa, Bott wrote a response [10], apologizing for accidentally omitting Shimura's role. He said he would gladly replace the offending sentences by "At Woods Hole Atiyah and I discovered how to generalize Shimura's conjectured fixed point formula to the elliptic context, and eventually we were able to establish this generalization by pseudo-differential techniques." The controversy did not end there. Shimura was not appeased, for Bott's apology was quite nuanced. Indeed, Bott went on to say that he had the impression of working on the holomorphic fixed point formula before meeting Shimura, so that he always 


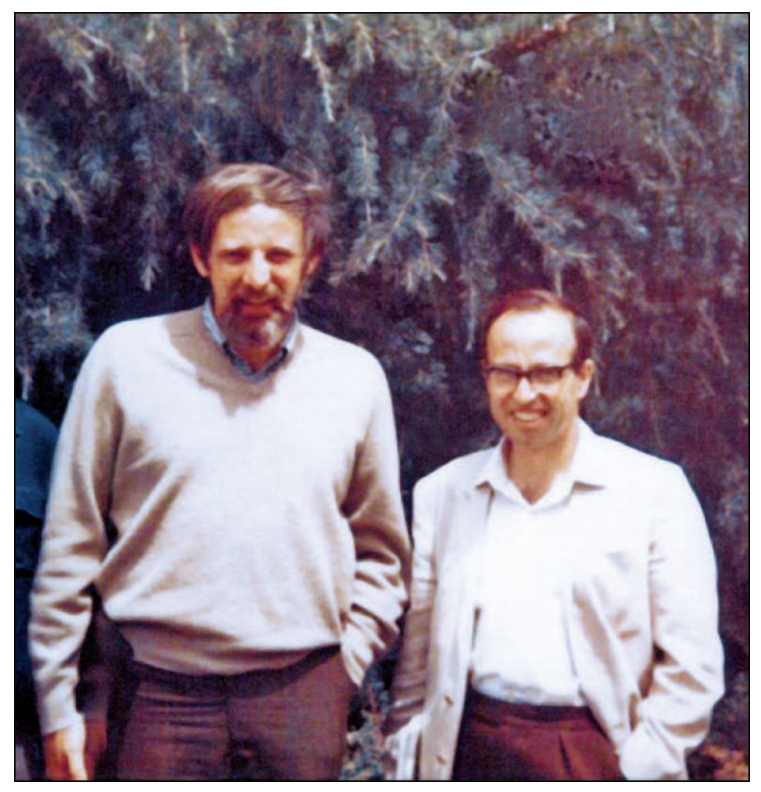

Figure 4. Raoul Bott and Michael Atiyah in the early 1970s.

remembered the encounter with Shimura "more as a confirmation than a revelation." Bott's statement raised some doubt about whether Shimura was solely responsible for the conjecture or whether Atiyah and Bott had some ideas of their own before meeting him.

\section{The Holomorphic Lefschetz Fixed Point Theorem}

A holomorphic map $f: M \rightarrow M$ from a complex manifold $M$ to itself induces a linear map $\left.f^{*}\right|_{H^{q}(M, \mathcal{O})}$ on the cohomology vector space $H^{q}(M, \mathcal{O})$, where $\mathcal{O}$ is the sheaf of holomorphic functions on $M$. The holomorphic Lefschetz number $L(f, \mathcal{O})$ of $f$ is defined to be the alternating sum $\left.\sum_{q}(-1)^{q} \operatorname{tr} f^{*}\right|_{H^{q}(M, \mathcal{O})}$ of the traces of the induced linear maps $\left.f^{*}\right|_{H^{q}(M, \mathcal{O})}$. Unlike the classical Lefschetz number $L(f)$, the holomorphic Lefschetz number is no longer necessarily an integer but can be any complex number. The holomorphic Lefschetz fixed point formula computes the holomorphic Lefschetz number $L(f, \mathcal{O})$ of a holomorphic self-map with isolated nondegenerate fixed points as a sum of contributions from the fixed points:

$$
L(f, \mathcal{O})=\sum_{f(P)=P} \frac{1}{\operatorname{det}\left(1-J(f)_{P}\right)},
$$

where $J(f)$ is the Jacobian matrix of $f$ with respect to any local holomorphic coordinate system. If $1 / \operatorname{det}\left(1-J(f)_{P}\right)$ is interpreted as the multiplicity of the fixed point $P$, then the holomorphic Lefschetz number also counts the number of fixed points of $f$, but, interestingly, the multiplicity of a fixed point need no longer be an integer!
In the example of the 2-sphere above, the 2sphere can be given a complex structure as $\mathbb{C} P^{1}$, the complex projective space of dimension 1 . The rotation map $f: \mathbb{C} P^{1} \rightarrow \mathbb{C} P^{1}$ is given in the finite plane $\mathbb{C}$ by $f(z)=e^{i \alpha} z$, so the Jacobian matrix of $f$ at the fixed point 0 is $e^{i \alpha}$. At the other fixed point $z=\infty$, a local coordinate is $w=1 / z$, and in terms of $w$, the map $f$ is

$$
w \mapsto \frac{1}{e^{i \alpha} \cdot \frac{1}{w}}=e^{-i \alpha} w,
$$

so the Jacobian matrix $J(f)$ at the fixed point $\infty$ is $e^{-i \alpha}$. The cohomology $H^{q}\left(\mathbb{C} P^{1}, \mathcal{O}\right)$ is $\mathbb{C}$ in dimension 0 , generated by the constant 1 , and 0 in all other dimensions. Therefore, the holomorphic Lefschetz number of $f$ is

$$
\begin{aligned}
L(f, \mathcal{O}) & =\left.\operatorname{tr} f^{*}\right|_{H^{0}\left(\mathbb{C} P^{1}, \mathcal{O}\right)}=1 \\
& =\frac{1}{1-e^{i \alpha}}+\frac{1}{1-e^{-i \alpha}},
\end{aligned}
$$

which verifies the holomorphic Lefschetz fixed point theorem in this case.

The holomorphic Lefschetz fixed point theorem also has some stunning consequences. For example, a complex algebraic variety $M$ is said to be rational if it is birational to a complex projective space $\mathbb{C} P^{n}$; i.e., there exist meromorphic maps $g: M \rightarrow \mathbb{C} P^{n}$ and $h: \mathbb{C} P^{n} \rightarrow M$ such that the composites $g \circ h$ and $h \circ g$ are the identity maps outside finitely many hypersurfaces. A compact smooth rational variety has the same cohomology $H^{q}(M, \mathcal{O})$ as $\mathbb{C} P^{n}$. Therefore, the holomorphic Lefschetz number of a holomorphic self-map of a compact smooth rational variety is 1 . By the holomorphic Lefschetz fixed point theorem, every holomorphic self-map of a compact smooth rational variety must have a fixed point. This is a holomorphic analog of the classical Brouwer fixed point theorem on a disk.

The holomorphic Lefschetz fixed point theorem can be generalized to holomorphic $p$-forms. On a complex manifold $M$ of complex dimension $n$, for $0 \leq p \leq n$, let $\Omega^{p}$ be the sheaf of holomorphic $p$-forms on $M$. For each $p$, one can define a holomorphic Lefschetz number

$$
L\left(f, \Omega^{p}\right)=\left.\sum_{q=0}^{n}(-1)^{q} \operatorname{tr} f^{*}\right|_{H^{q}\left(M, \Omega^{p}\right)} .
$$

This holomorphic Lefschetz number $L\left(f, \Omega^{p}\right)$ is also a sum of contributions from the fixed points. The precise form of the contribution is given by the Woods Hole fixed point theorem.

\section{Shimura's Conjecture at Woods Hole}

Fortunately, we have two independent accounts of the Woods Hole fixed point theorem from the time of the conference: the report of Atiyah and Bott on the Woods Hole Fixed Point Theorem Seminar [4], which was distributed at the conference in 1964, 
and J.-P. Serre's letter to Alexander Grothendieck dated August 2-3, 1964 [18]. The Atiyah-Bott report has never been published but will be included in the forthcoming Volume 5 of Bott's Collected Papers.

In the Atiyah-Bott report, one finds a precise statement of Shimura's conjecture:

"Suppose $X$ is a nonsingular projective algebraic variety over an algebraically closed field $k$ and that $f: X \rightarrow X$ is a morphism of $X$ into itself. Suppose further that $E$ is a vector bundle over $X$ and that $f$ admits a lifting $\phi$ to $E$ - that is, a vector bundle map $\phi: f^{-1}(E) \rightarrow E$. Such a lifting then defines in a natural way an endomorphism $(f, \phi)^{*}$ of the cohomology vector spaces $H^{*}(X, \mathcal{E})$ of $X$ with coefficients in the locally free sheaf $\mathcal{E}$ of germs of sections of $E$, and we may therefore form the Lefschetz number of this endomorphism:

$$
\chi(f, \phi, E)=\sum_{q}(-1)^{q} \operatorname{tr}\left\{\left.(f, \phi)^{*}\right|_{H^{q}(X ; \mathcal{E})}\right\} .
$$

“...Finally note that, at a fixed point $p$, the lifting $\phi$ determines an endomorphism $\phi_{p}$ of (the fiber) $E_{p}=E_{f(p)}$ and so has a well-determined trace.

"With this understood, the Shimura conjecture, which we now propose to call the Woods Hole fixed point theorem, is given by the relation:

$$
\chi(f, \phi, E)=\sum_{p} \frac{\operatorname{tr} \phi_{p}}{\operatorname{det}\left(1-d f_{p}\right)},
$$

where $p$ runs over the fixed points of $f . "$

Serre's letter to Grothendieck of August 2-3, 1964 , says essentially the same thing but in French.

Depending on the context, there are many Lefschetz fixed point theorems-for smooth maps, holomorphic maps, sheaves of holomorphic forms, vector bundles, elliptic complexes, and étale cohomology-and they are not exactly the same, although some are special cases of others. In the literature there seems to be a common understanding of the Woods Hole fixed point theorem to mean the Lefschetz fixed point theorem for vector bundles, which was what Shimura conjectured.

From this point of view, what Bott said in the 2001 Notices interview was perfectly accurate and true: he and Atiyah discovered at Woods Hole the Lefschetz fixed point formula for elliptic complexes. Of course, its inspiration was Shimura's conjecture on vector bundles. It took a great leap of imagination to go from vector bundles to elliptic complexes. For this, and for his other work, Atiyah was justly awarded the Fields Medal.

The Atiyah-Bott report also states that the algebraic case of Shimura's conjecture was proven at the conference through the collective effort of a large number of participants, "especially that of Verdier, Mumford, and Hartshorne,..., along more or less classical lines from the Grothendieck version of Serre duality." Serre reports in his letter to Grothendieck that Atiyah gave an algebraic proof of Shimura's conjecture using duality and local Ext and that Bott gave a complex analytic proof using differential forms.

\section{Atiyah and Bott's Acknowledgments}

In several contemporaneous published accounts of their work on the Lefschetz fixed point formula, Atiyah and Bott acknowledged the contribution of Shimura.

In the introduction to their 1964 Harvard notes [5], Atiyah and Bott wrote, "Our main formula also generalizes a result of Eichler on algebraic curves which was brought to our attention by Shimura during the recent conference at Woods Hole on algebraic geometry. In fact, this work resulted precisely from our attempt to prove Shimura's conjectures in this direction."

In the introduction to the 1966 Bulletin article [6, p. 245], Atiyah and Bott wrote, "Taking the classical operators of complex and Riemannian geometry we obtain a number of important special cases (Theorems 2,3). The first of these was conjectured to us by Shimura and was proved by Eichler for dimension one." By "the first of these" they meant Theorem 2 [6, p. 247], which is the Lefschetz fixed point formula for holomorphic vector bundles.

In the two Annals of Mathematics papers ([7] and [8]) in 1967 and 1968, although no mention was made of Shimura in the introduction, under the holomorphic Lefschetz fixed point formula [8, p. 458], Atiyah and Bott wrote, "To recapitulate: for a transversal endomorphism $f$ of a complex analytic manifold $X$, our fixed point formula specializes to

(1) $L\left(f^{p, *}\right)=\sum_{f(P)=P} \operatorname{trace}_{\mathbb{C}}\left(\lambda^{p} d f_{P}\right) / \operatorname{det} \mathbb{C}\left(1-d f_{P}\right)$

where

$$
L\left(f^{p, *}\right)=\sum(-1)^{q} \operatorname{trace} H^{p, q}(f) .
$$

"It is this formula which Shimura conjectured during a conference at Woods Hole in 1965 [sic], and which furnished the impetus for this work. For curves (4.9) had already been established by Eichler in [11]. Shimura and Eichler were of course thinking in the framework of algebraic geometry. There it turned out that the full duality theory of Serre and Grothendieck yields this result even over arbitrary characteristic."

These accounts seem to establish unequivocally that Shimura was the originator of the conjecture on the holomorphic fixed point formula, with the numerator and denominator as stated.

\section{Shimura's Forgotten Conjecture}

According to Shimura ([16, p. 131], [17]), at Woods Hole he had conjectured much more than the Lefschetz fixed point formula for holomorphic maps. 


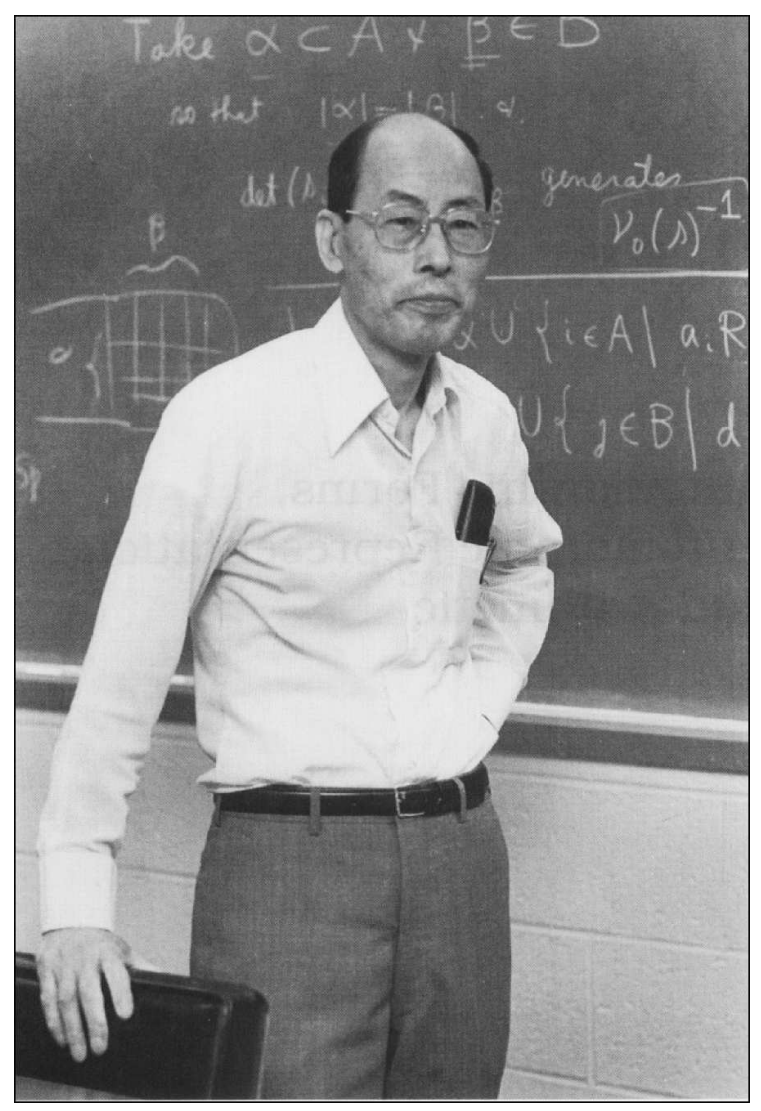

Figure 5. Goro Shimura in May 1996.

A smooth correspondence of a manifold $M$ is a submanifold of the same dimension as $M$ in $M \times M$. The graph of a map from $M$ to $M$ is a special case of a correspondence that satisfies the vertical line test. Shimura said he was interested at the time in Eichler's formula for the Hecke correspondence in dimension one and he had made a conjecture for an algebraic correspondence that would generalize Eichler's formula to higher dimensions. In his own words, "My intention was to extend Eichler's result to higher-dimensional cases. Therefore, my conjecturing only the map-case would have been ridiculous" [17]. When the algebraic correspondence is a holomorphic map, Shimura's conjecture would specialize to the holomorphic fixed point theorem, and this was what Atiyah and Bott proved. Shimura's recollection, however, is not supported by either the Atiyah-Bott report [4] or Serre's letter [18].

Although I have not been able to locate any reference in the literature, Shimura thinks that the Lefschetz fixed point theorem for a smooth correspondence is known but that the algebraic case remains open. Regrettably, no one seems to have written down Shimura's conjecture of a Lefschetz fixed point theorem for an algebraic correspondence, and Shimura no longer remembers its exact formulation, and so it appears that this

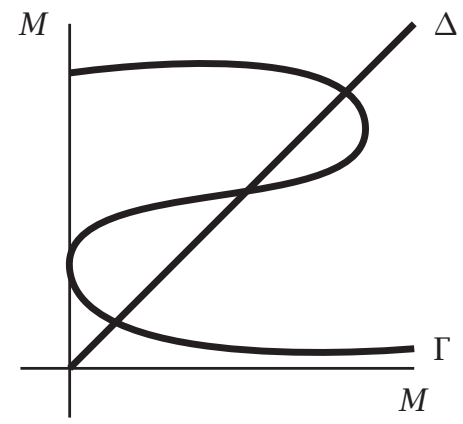

Figure 6. A transversal intersection of a correspondence $\Gamma$ with the diagonal.

fascinating conjecture that would simultaneously generalize the holomorphic fixed point theorem and Eichler formula for the Hecke correspondence is now lost.

\section{Recollections}

What about Bott's recollection that he and Atiyah had worked on the formula before meeting Shimura? With his characteristic self-deprecating sense of humor, Bott wrote in [11], "Actually I seem to recall that Atiyah and I stumbled on some version of this formula ourselves, but as no one else is able to confirm this fact, I now have to attribute it to the self-aggrandizing instincts of memory." In the commentary to his Collected Works [1], Atiyah wrote in 1988, "At the Woods Hole conference in the summer of 1964 Bott and I learnt of a conjecture of Shimura concerning a generalization of the Lefschetz fixed-point formula for holomorphic maps. After much effort we convinced ourselves that there should be a general formula for this type for maps preserving any elliptic operator (or more generally any elliptic complex)." More recently, in 2013, Atiyah sent me an email message [2] stating, "I am afraid that Raoul is to blame for suggesting that $A B$ [Atiyah and Bott] were already working on the problem before we met Shimura. I never supported this view."

Supposing Bott's recollection to be correct, a possible explanation is that Shimura had told his conjecture to several people at the conference, who then told it to Atiyah and Bott, so that without Bott's realizing it, Shimura was the ultimate source of the conjecture on the holomorphic Lefschetz fixed point formula. Indeed, Shimura wrote in [17], "During the conference, I recalled this conjectural formula, and told it to John Tate, whom I knew since 1958. Then Tate told it to Atiyah and Bott. Eventually I discussed the matter with them."

As for the anecdote about number theorists telling Atiyah and Bott that they were wrong, it is unfortunate that Bott used the phrase "the number theorists" when he meant only the number theorists they consulted, for the former could 
mean all the number theorists at Woods Hole in 1964, including Shimura. On this point, Atiyah elaborated in a message [3] to me in 2013:

"I insist on the accuracy of my memory (and that of Raoul). We definitely did ask Tate and Cassels to check an example in dimension 1 (to do with elliptic curves having complex multiplication, I believe). They came back and told us it did not work. But we felt it was too beautiful not to be true and so we persevered. Realizing that the Hermann Weyl formula came out as a special case, we became ever more convinced. This episode played such an important part in the story that I cannot be mistaken.

Unfortunately, neither Tate nor Cassels remembers the incident, but for them it was a minor story not worth remembering. For me it was crucial.

How does one reconcile this with the statement of Shimura that Eichler had proved the general HLF [holomorphic Lefschetz formula] in dimension 1 ? My best guess is that Eichler only studied the case when the line bundle was a power of the canonical bundle, since this is what enters into modular forms, and all Eichler's work was concerned with this. Shimura conceded as much in one of his emails to me but then seemed to retract.

So my version is that Shimura may indeed have conjectured HLF in general (but it is frustrating that he never wrote it down and now claims that he cannot remember exactly what the conjecture was). But Eichler's work in dimension 1 was not conclusive and there was no serious evidence for the general HLF (before the Weyl formula). Our purpose in asking the number theorists was to check more cases."

John Tate wrote in July 2013 [20], “I don't remember Shimura's telling me about the formula. In fact I don't remember anything about that formula at the conference. But it was forty-nine years ago, and memories fade, especially if they are about a subject like several complex variables or elliptic PDE's that I didn't know much about. I wonder why he would tell me. It's true that I had met him in Paris in spring of 1958, but we did not know each other well at all." Also in [19], "I have no memory of being asked with Cassels to check something about elliptic curves. I think Raoul might have been exaggerating something to make a good story there...."

\section{Conclusions}

In the intervening years since 1964, Shimura's pivotal role in the genesis of the Woods Hole fixed point theorem has largely been forgotten. For instance, in Griffiths and Harris's widely used Principles of Algebraic Geometry [12], there is a discussion of the holomorphic Lefschetz theorem and a reference to Atiyah and Bott's Annals paper [8], but no mention of Shimura. Similarly, in various research articles on the holomorphic fixed point formula ([14], [21], [22]) there is no mention of
Shimura. It may be that his conjecture, at least the one for holomorphic maps, had such a short life that it never really registered in the public's consciousness.

In his autobiography [16, p. 131], Shimura wrote with regard to the Woods Hole fixed point theorem, "But interestingly they [Atiyah and Bott] gradually tried to minimize my contribution." Insofar as this implies intentional deeds on the part of Atiyah and Bott, I can find no evidence that this is so. Except for Bott's Notices interview in 2001, in which he accidentally omitted Shimura's role and for which he later apologized publicly, in every published account of the Woods Hole fixed point theorem, Atiyah and Bott have always acknowledged Shimura's conjecture. As late as 2004, one year before he passed away, as if to compensate for the 2001 Notices interview, Bott mentioned "Shimura's formula" or "Shimura's conjecture" no fewer than six times in a short article on the history of the Woods Hole fixed point formula [11]. Over the thirty years that I knew Raoul Bott, he always told me to be generous with credit. It was not in his character to deny credit where it was due.

The preponderance of the evidence suggests to me that Shimura and Shimura alone was responsible for the original conjecture on the holomorphic Lefschetz fixed point formula, which Atiyah and Bott proved and generalized to elliptic complexes during the Woods Hole conference in 1964. It is possible that Shimura had made a more general conjecture on algebraic correspondences which no one remembers anymore.

\section{Postscript}

Both Michael Atiyah and Goro Shimura have read this article. Atiyah said, "I think it is very fair," but Shimura declined to endorse it.

\section{Acknowledgments, Photo Sources and Credits}

I am grateful to Michael Atiyah, Jeffrey D. Carlson, William Fulton, Allen Hatcher, George Leger, Winnie Li, Steve Schnably, Goro Shimura, Jacob Sturm, John Tate, and four anonymous reviewers for The Notices for helpful comments and discussions. Figure 4: from the Bott Family Collection.

Figure 5: from Automorphic Forms, Automorphic Representations and Arithmetic, Proceedings of Symposia in Pure Mathematics, Vol. 66, Part 2, page iv, edited by Robert S. Doran, Ze-Li Dou, and George T. Gilbert, Amer. Math. Soc., Providence, RI, 1999.

\section{References}

[1] M. F. Atiyah, Commentary, Collected Works, vol. 3, Oxford University Press, 1988, pp. 2-3.

[2] _ _ email message to Loring Tu, June 23, 2013.

[3] _ email message to Loring Tu, July 7, 2013. 
EXCEPTIONAL

\section{Pure}

Mathematics

Research

\section{COMPLIMENTARY ACCESS TO OVER 100 ARTICLES IN NOVEMBER AND DECEMBER}

\section{Read the latest papers in journals including:}

- Bulletin of the Australian Mathematical Society

- Compositio Mathematica

- Glasgow Mathematical Journal

- Journal of the Institute of Mathematics of Jussieu

- Mathematical Proceedings of the Cambridge Philosophical Society

- Proceedings of the Edinburgh Mathematical Society

- The Review of Symbolic Logic

\section{journals.cambridge.org/pm15}

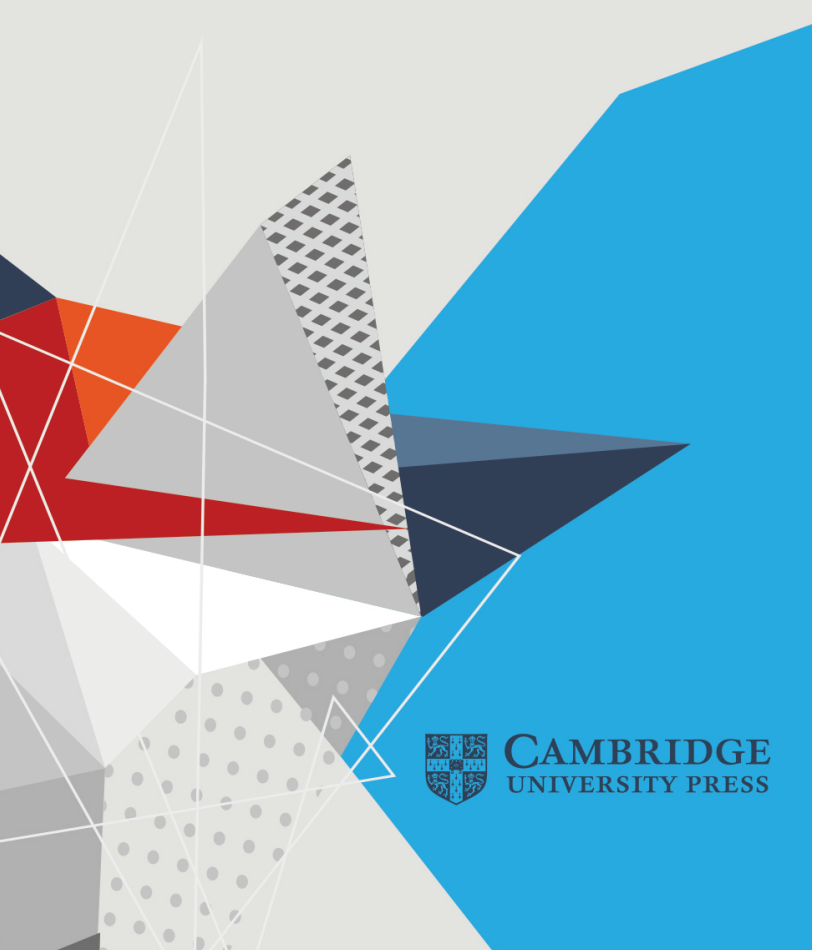

[4] M. F. AtiYAH and R. BotT, Report on the Woods Hole fixed point seminar, 1964, in Raoul Bott, Collected Papers, Volume 5, Loring Tu, editor, Springer, Basel, to appear.

[5] Notes on the Lefschetz fixed point theorem for elliptic complexes, mimeographed notes, Harvard University, Fall 1964.

[6] _ A Lefschetz fixed point formula for elliptic differential operators, Bull. Amer. Math. Soc. 72 (1966), 245-250.

[7] _ A Lefschetz fixed point theorem for elliptic complexes: I, Annals of Math. 86 (1967), 374-407.

[8] _ A Lefschetz fixed point theorem for elliptic complexes: II. Applications, Annals of Math. $\mathbf{8 8}$ (1968), 451-491.

[9] A. Jackson, Interview with Raoul Bott, Notices Amer. Math. Soc. 48 (2001), no. 4, 374-382.

[10] R. BoTT, Response to Shimura's Letter, Notices Amer. Math. Soc. 48 (2001), no. 7, 678-679.

[11] _ Introduction, in Woods Hole Mathematics: Perspectives in Mathematics and Physics, edited by N. Tongring and R. C. Penner, Series on Knots and Everything, vol. 34, World Scientific Publishing, Hackensack, NJ, 2004, pp. vii-xi.

[12] P. GRIFFITHS and J. HARRIS, Principles of Algebraic Geometry, John Wiley \& Sons, New York, 1978.

[13] A. GRothendiecK, SGA 5: Cohomologie $\ell$-adique et fonctions $L$, Lecture Notes in Math. vol. 589, Springer, New York, 1977.

[14] V. K. PATOdI, Holomorphic Lefschetz fixed point formula, Bulletin Amer. Math. Soc. 79 (1973), $825-828$.

[15] G. ShImura, History of the Woods Hole fixed-point theorem, Letters to the Editor, Notices Amer. Math Soc. 48 (2001), no. 7, 678 .

[16] _ , The Map of My Life, Springer, New York, 2008.

[17] C Commentary on the Woods Hole fixed point formula, email message to Loring Tu, June 16, 2013.

[18] J.-P. Serre, Letter to A. Grothendieck, August 23, 1964, in Correspondance Grothendieck-Serre, P. Colmez and J.-P. Serre, editors, Société Mathématique de France, Paris, 2001, pp. 149-164.

[19] J. TATE, email message to Loring Tu, July 9, 2013.

[20] _ email message to Loring Tu, July 12, 2013.

[21] D. TOLEDO, On the Atiyah-Bott formula for isolated fixed points, J. Differential Geom. 8 (1973), 401-436.

[22] Y. L. L. ToNG, De Rham's integrals and Lefschetz fixed point formula for $d^{\prime \prime}$ cohomology, Bull. Amer. Math. Soc. 78 (1972), 420-422.

[23] J.-L. VERDIER, The Lefschetz fixed point formula in étale cohomology, Proc. Conf. Local Fields (Driebergen, 1966), Springer, Berlin, 1967, pp. 199-214. 